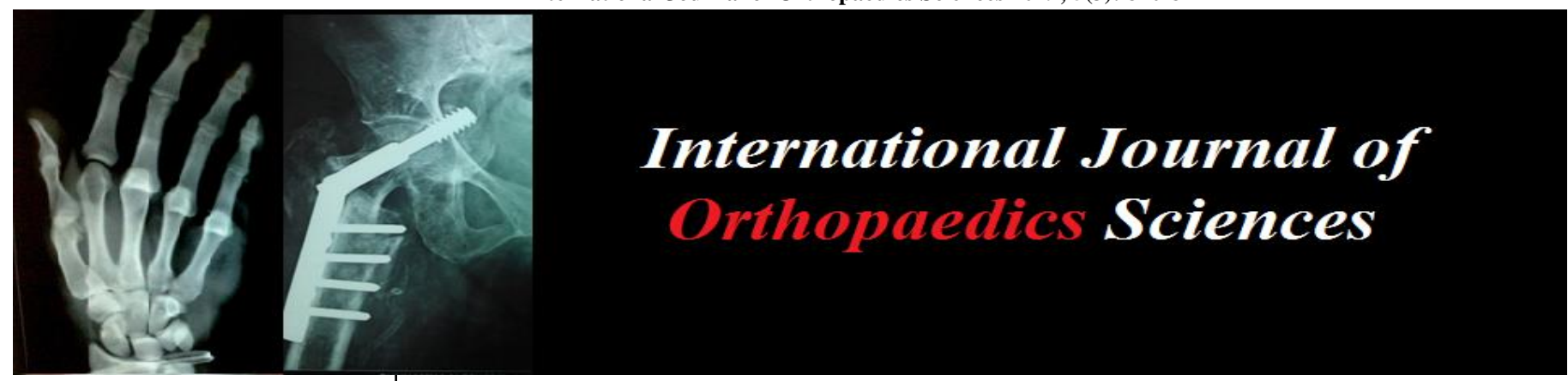

ISSN: $2395-1958$

IJOS 2019; 5(3): 810-814

(C) 2019 IJOS

www.orthopaper.com

Received: 14-05-2019

Accepted: 18-06-2019

Dr. Shenoy Ravindra

Yenepoya Medical College

Hospital, Karnataka, India

Dr. Ahmed Afroz

Yenepoya Medical College

Hospital, Karnataka, India

Correspondence

Dr. Ahmed Afroz

Yenepoya Medical College

Hospital, Karnataka, India

\section{Results of customized approach for giant cell tumour}

\author{
Dr. Shenoy Ravindra Mohan and Dr. Ahmed Afroz
}

DOI: $\underline{\text { https://doi.org/10.22271/ortho.2019.v5.i3n.1631 }}$

\section{Abstract}

Giant cell tumour is one of the commonest bone tumours encountered. They are usually benign, but locally aggressive and may occasionally undergo malignant transformation. Total excision and reconstruction is the treatment of choice. Based on this principle several methods are employed in treatment depending on the age, site and nature of the tumour. Choosing a proper method/s (Customization) is essential for good functional results. Herewith are presented three cases of Giant Cell Tumour, two arising from the proximal tibia and one from the distal ulna, which were treated with customized approach, giving good results. The rationales behind choosing the treatment method/s have been discussed.

Keywords: Giant cell tumour, customized treatment, zoledronic acid, bone cement, inferior radio-ulnar

\section{Introduction}

Giant-cell tumor (GCT) is a benign but locally aggressive tumor. It accounts for 5\% of all primary bone tumours ${ }^{[1]}$. Its peak incidence is between 20-40 years with a slight female predominance ${ }^{[2]}$. Giant cell tumours have a high local recurrence rate of $20 \%$ to $50 \%$ of which $10 \%$ may undergo malignant transformation at recurrence and $1 \%$ to $4 \%$ may give pulmonary metastases ${ }^{[3]}$. GCT is predominantly epiphyseal tumour, more commonly occurring in the distal femur, proximal tibia, and distal radius. GCTs of distal end of the ulna are extremely rare and accounts for $0.45 \%$ to $3.2 \%$ of all the cases ${ }^{[4]}$.

The main clinical symptoms include local swelling, warmth, and pain of the affected limb. Radiologically, it appears as a pure lytic cystic lesion, which is located in the epiphysis, growing eccentrically with thinning of the cortex ${ }^{[3]}$. Pathological fracture is a late complication.

The treatment for GCT is total excision of tumour tissue and reconstruction of the defect. Extended curettage and filling the cavity with bone grafts with or without bone cement (polymethyl methacrylate) or bone graft substitutes form the mainstay of the treatment. High local recurrences of about $>50 \%$ are reported after simple curettage without the use of adjuvants and upto $25 \%$ with the use of adjuvants such as phenol, hydrogen peroxide $\left(\mathrm{H}_{2} \mathrm{O}_{2}\right)$, liquid nitrogen. High-speed burring, and thermo-coagulation are also being used to decrease the recurrence ${ }^{[2]}$. In locally extensive disease where curettage is not feasible, enbloc resection and arthrodesis by turn plasty or rotation plasty or arthroplasty using mega prosthesis is advocated. However, they are not primarily used as first line of treatment when resection and bone grafting is feasible. Treatment should be aimed at biological restoration of joint.

Zoledronic acid belongs to bisphosphonate group of drugs (1-Hydroxy-2-imidazol -1-ylphosphonoethyl), has been used in treatment of GCT. It acts by activating protein kinase C which inhibits osteoclastic mediated bone resorption and induces antiproliferative activity along with apoptotic effect ${ }^{[2]}$. Zoledronic acid is used as neoadjuvant or adjuvant chemotherapeutic agent. Some authors even recommend use of zoledronic acid in bone cement to prevent recurrence ${ }^{[2,17,18]}$.

As an alternative to bisphosphonates, Denosumab is used in the treatment for GCT in unresectable cases. It is a monoclonal antibody against RANK-L, expressed in the tumour cells. Its use is limited considering its high cost, local and systemic side effects. 


\section{Subjects and Methods}

Between June 2017 and October 2017, three patients diagnosed with GCT, two around the knee joint (Proximal tibia) and one from distal ulna underwent customized treatment. Evaluation of bone destruction through radiographs and magnetic resonance imaging was of paramount importance to define surgical procedure. The diagnosis of the lesions was confirmed by percutaneous biopsy using a Jamshidi needle. The entire tumour mass was sent for histopathology after excision and additional sample was taken from the margins to check for clearance.

The first case, GCT of proximal tibia with a pathological fracture (Fig 1) underwent treatment with Zoledronic acid for 6 monthly cycles prior to extended curettage, hydrogen peroxide wash, bone grafting and cementing. The second case, GCT of distal ulna (Fig 2) underwent enbloc excision and reconstruction of inferior radio-ulnar joint using a tricortical iliac crest autograft replacing the head of ulna. The ulnar collateral ligament was anchored to the graft. Third case, GCT of proximal tibia (Fig 3) underwent extended curettage and bone grafting along with bone graft substitutes. Post operatively, patient received Zoledronic acid as his margin showed presence of giant cells.

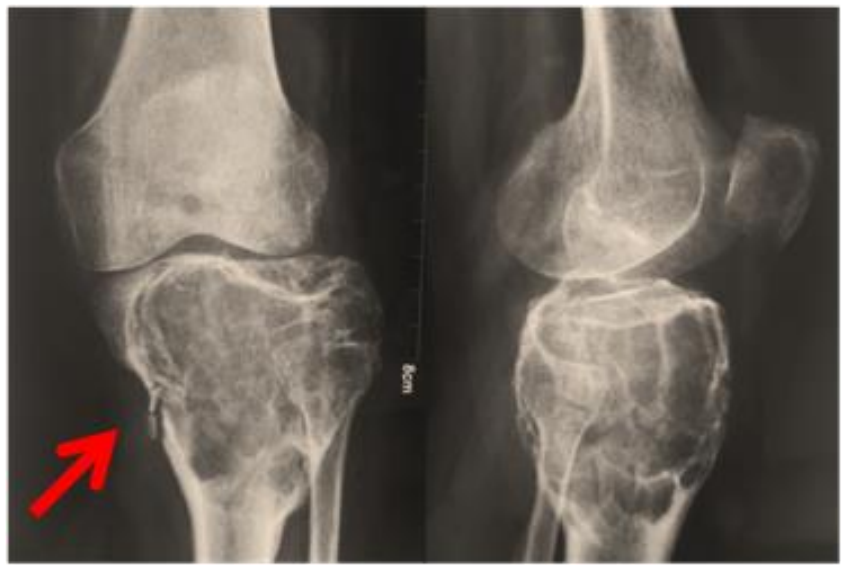

Fig 1: Case one at the time of presentation with pathological fracture (red arrow) and thinning of the cortex
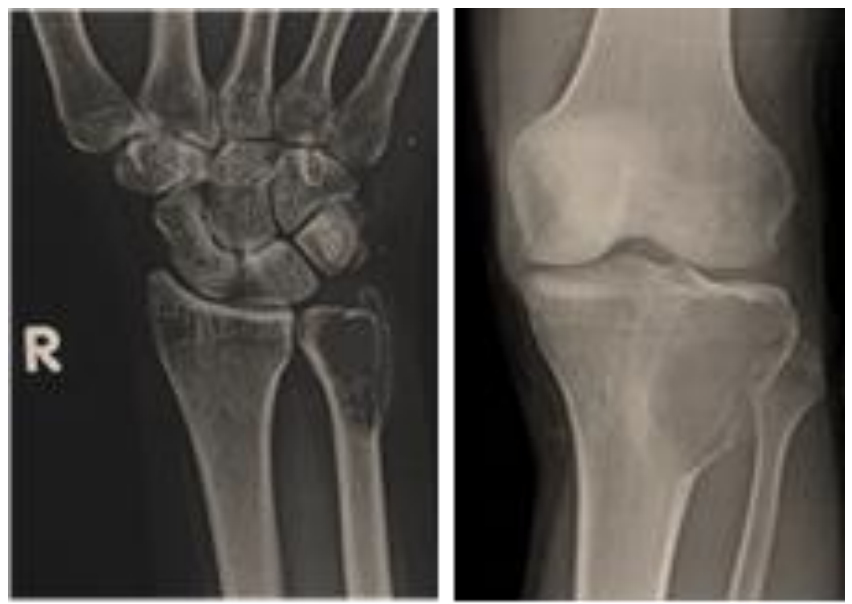

Fig 2, 3: Case 2 (left) and case 3 (right) respectively

Patients were evaluated every 15 days in the first month, followed by monthly follow-up appointments up to the 6 months, and then follow-up appointments every three months until one year of surgery. Patients who did not present relapse in the first years after surgery were considered cured. However, annual follow-up lifelong continues. Case one was followed for 12 months, Case two for over two years and Case three for 18 months.

\section{Results}

All the patients tolerated the treatment well. No side effects with use of Zoledronic acid were observed. No patients complained of pain after 1 month post-surgery and were able to get back to their work. Patients with GCT of tibia started full weight bearing by the end of 2 months and have full range of movement. Case two, GCT of distal ulna has full supination and pronation and has no difficulty in doing his work.

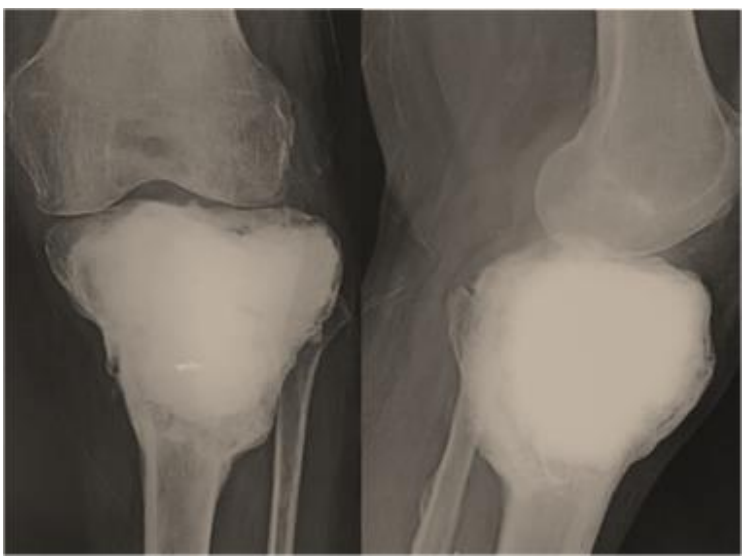

Fig 4: One year follow up of Case one

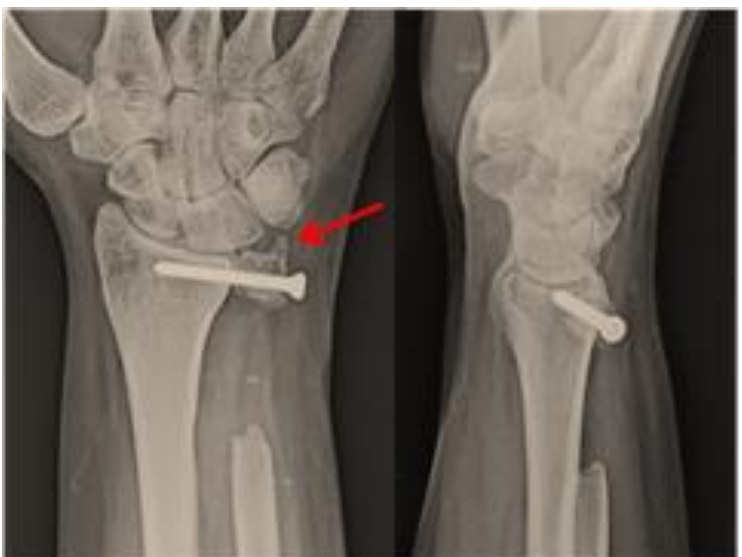

Fig 5: Case two follow-up x ray showing re-formation of ulnar styloid process (red arrow)

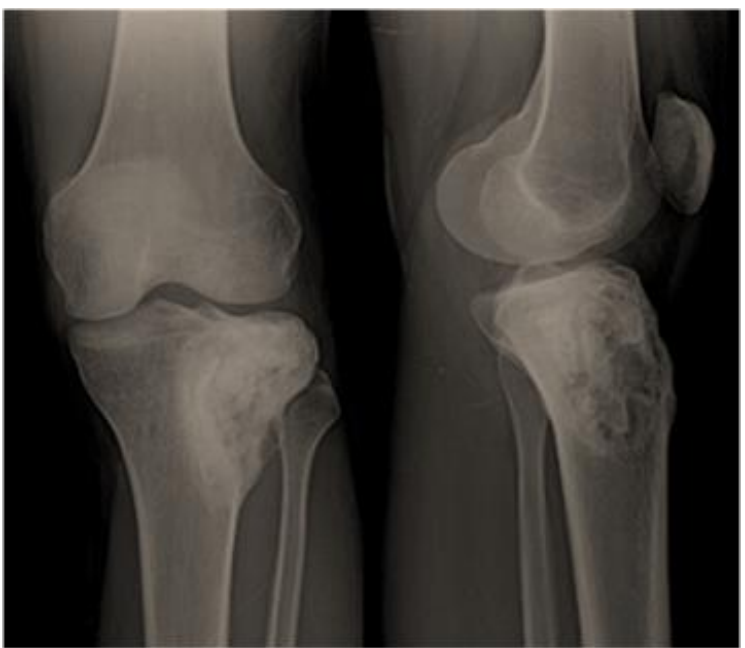

Fig 6: Case 3 follow up at year showing consolidation 
Patients were subjected to radiographs on follow up. None of them showed signs of recurrence at the end of one year. Radiograph of case 2 at the end of 9 months showed the graft in place and formation of ulnar styloid process as a result of anchoring the ulnar collateral ligament to it (Fig 5). In Case 3, initial follow up radiographs revealed resolution of the bone graft substitutes and subsequent radiographs showed consolidation (Fig 6). No evidence of recurrence seen in radiographs at follow-up.

\section{Discussion}

GCT is a benign tumor but has the potential for local aggression, recurrence, and occasional lung metastases ${ }^{[5]}$. This tumor does not remain latent and a small lesion tends to evolve and lead to progressive destruction of the bone. Therefore, surgical treatment is indicated at the earliest. The principle behind the treatment is total excision of the tumor and reconstruction.

Curettage and clearance assisted by adjuvant methods such as chemical cauterization, liquid nitrogen etc has been defined as the preferred method of treatment for most cases of GCT, as functional outcome is better and recurrence rate reduces [11, ${ }^{12]}$. Wide resection has the advantage of lower recurrence, as it removes the tumor entirely and it is preferred in locally advanced GCT with bone destruction ${ }^{[5,11]}$. In such a cases, reconstruction becomes a challenging task especially when the tumor is huge and located around the knee joint. Surgeons in the past have resorted to techniques such as rotation plasty and turn plasty after en-bloc excision of the tumor but it's a highly morbid procedure and has its own complications such as shortening of limb, loss of knee movement etc. Megaprosthesis solves the above problems by replacing the knee joint but has its own disadvantages such as loosening, breakage etc which necessitates a second surgery. Longevity of the mega-prosthesis is questionable. Further, it gives only a limited range of movements. Hence, is undesirable and generally is reserved for recurrent cases and cases which are not amenable for other methods of treatment. Thus, it is reasonable not to consider that mega-prosthesis as a primary method for treating a GCT.

In this study, Case one - GCT of proximal tibia was not amenable for curettage and bone grafting/cementing as the cortex had thinned out and there was a pathological fracture. The breech in cortex did not allow impaction of bone grafts or the bone cement. The other option was en-bloc excision and use of mega-prosthesis, which is a highly morbid procedure leading to considerable loss of normal bone and muscle attachments. Patient will have limited functionality at the knee joint. The next option is arthrodesis by turn plasty where proximal tibia is excised in total and the medial half of distal femur split and turned $180^{\circ}$ and fixed to tibia distally and condyle of femur proximally. The joint is arthrodesised. This ensures complete removal of the tumour at the cost of function. This is a procedure of the past and perhaps to be considered when nothing else is possible.

Hence, the treatment was customised by attempting to shrink the size of the tumour first by giving Zoledronic acid and then plan for definitive surgery (i.e. excision by curettage with bone grafting and other filling materials like bone cement etc). Patient was given 6 cycles of Zoledronic acid $(4 \mathrm{mg})$ at monthly intervals which resulted in shrinkage of the tumor and consolidation of the margins (Fig 7). Then, as planned patient underwent extended curettage followed by thorough hydrogen peroxide wash. Bone cement was impacted into the cavity after plugging the medullary canal and distal cavity with cortico-cancellous bone grafts harvested from iliac crest. Gradual weight bearing was started after 2 months post operatively and patient had excellent outcome by the end of 6 months. The Scandinavian Sarcoma Group, in their multicentric study by Kivioja et al. involving 294 patients, reported that filling the cavity with bone cement was shown to be a prognostic factor and the recurrence rate was 0.20 for filling with cement and 0.56 for intralesional surgery without cementation with $\mathrm{p}=0.001^{[6]}$.

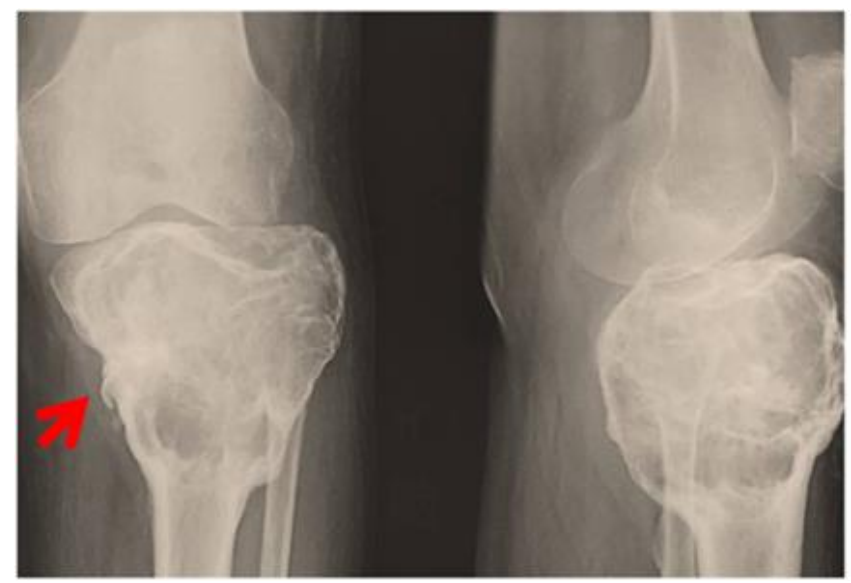

Fig 7: Case one after 6 monthly cycles of Zoledronic acid showing shrinkage of tumour size and consolidation of tumour margins (red arrow represents the site of pathological fracture, which after surgery is well consolidated).

Patients affected by GCT are usually young, and therefore there is a concern to preserve the joint and avoid possible early arthroplasty. Joint replacement by endo-prosthesis brings some disadvantages, such as infection and the risk of successive revisions ${ }^{[5]}$. Thus, many studies indicate a preference for biological reconstruction methods in the treatment of GCT, in the hope that primary procedure will be definitive. Use of bone grafts from bone bank is also preferred for reconstruction of larger defects. The use of bone cement is a well-established method that presents good long-term results. Retrospective study of 46 cases of GCT undergoing curettage and cementation published by Baptista et al. ${ }^{[13]}$, concluded that the distance from cement to subchondral bone has a prognostic relationship to the development of osteoarthritis, but not to final functional outcome of the patient.

In the Second case - GCT affecting the distal ulna, patient underwent en-bloc excision and reconstruction of inferior radioulnar joint using a tricortical iliac crest autograft. The ulnar collateral ligament was sutured to the graft. Resection of the distal ulnar end is most commonly performed procedure for disorders of the distal radioulnar joint ${ }^{[8]}$. Long-term results of the resection of distal ulna are not predictable, and instability usually develops within a year ${ }^{[9,15]}$. Goal in young and active patients is to preserve wrist stability with as much movement as possible. Hashizume et al. used a longer iliac bone graft to achieve the buttress effect against axial stress loading to reconstruct the inferior radio-ulnar joint after excision of distal ulna ${ }^{[10]}$. The procedure adopted here is a modification of the above said procedure where the ulnar collateral ligament was anchored to the iliac crest autograft (Fig 8). 


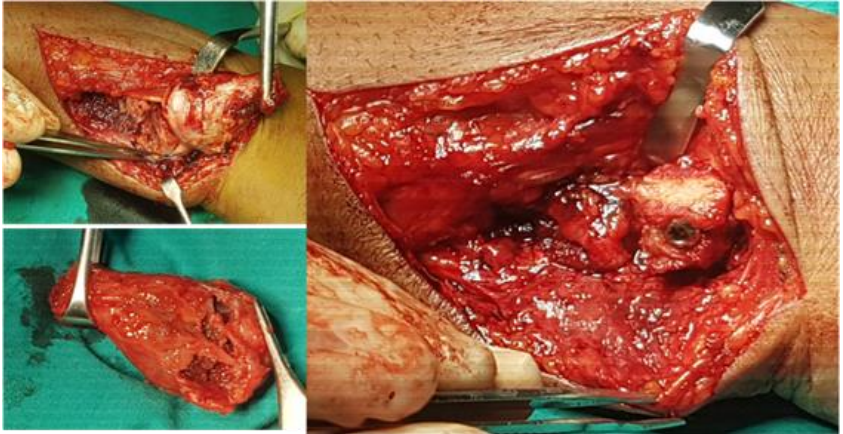

Fig 8: Intra operative pictures of case-2 showing en-bloc excision and reconstruction using a tricortical iliac crest autograft.

The procedure of intralesional excision and bone grafting was clearly inappropriate in this case as there was cortical breech, confirmed by MRI as well as intra-operatively. En-bloc excision of the distal ulna is curative in most of the cases but it has disadvantage of developing wrist instability with dynamic radioulnar convergence. This leads to wrist pain, weakness, and loss of grip strength as the ulnar stump may impinge upon the distal radius [15, 16]. Troublesome subluxation is one of the complications. Distal ulna prevents convergence and also ulna carries about $20 \%$ of the load of the hand ${ }^{[16]}$. Loss of the distal ulna has been shown to cause radiocarpal instability and even rupture of digital extensor tendons ${ }^{[1]}$. In order to maintain wrist stability following excision, reconstruction is recommended. Various methods have been described from biological reconstruction to endoprosthesis. High cost of implant and risk of loosening of implant hinder the use of endo-prosthesis. In the case presented tricortical iliac crest autograft was used to reconstruct. The results were satisfying. Patient has full range of movements and good grip strength (Fig 9).

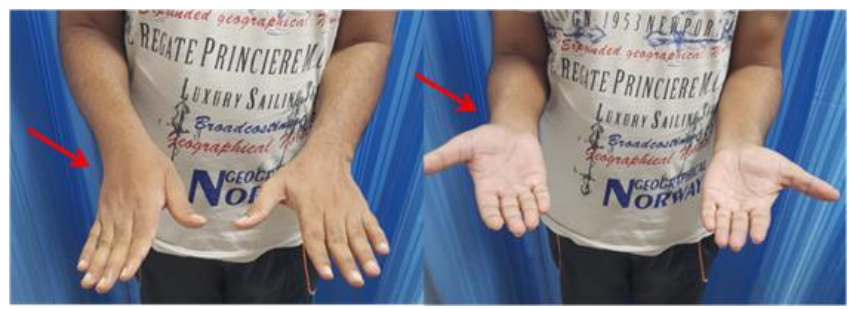

Fig 9: Functional outcome of case 2, full pronation and supination (Right hand - marked with red arrow)

The incidence of GCT recurrence varies in the literature. Dahlin et al. ${ }^{[14]}$ published a study with $60 \%$ of local recurrence in GCT patients who underwent curettage and grafting and recommended a more aggressive resection for local control. Retrospective study of forty four patients with GCT who underwent intralesional curettage with cementation or biological reconstruction, of which 24 patients received bisphosphonates peri-operatively, published by Zile Singh Kundu et al. ${ }^{[2]}$ concluded that the effect of reduction of local recurrence was significant with use of bisphosphonates and they recommended its use as an adjuvant therapy for giant cell tumor. In this study, Case-3 showed presence of tumor cells from the biopsy taken from margin after clearance. Hence, patient was treated with monthly cycles of Zoledronic acid (4mg slow IV) for three consecutive months. There was no evidence of recurrence clinically or radiologically and patient was symptom free when reviewed at the end of one year.

\section{Conclusion}

The treatment of GCT is still very challenging. We feel that the best treatment method for treatment of giant cell tumour is to preserve the joint and restore its function. Biological procedures should be preferred before contemplating on metallurgical solutions. Use of adjuvants such as hydrogen peroxide, phenol, liquid nitrogen, high speed burs for curetting the walls of cavity, thermo coagulation etc. can help to reduce the recurrence rates. Zoledronic Acid can be used in locally advanced GCT to shrink the tumour size, consolidate the margins and even following the surgical procedure to prevent the recurrence. We conclude that every case is different and needs customized treatment keeping the standard treatment protocol in mind i.e. total excision and reconstruction.

\section{Conflicts of interest}

The authors declare no conflicts of interest.

\section{Footnotes}

This study was conducted in Yenepoya Medical College Hospital, Mangalore, India.

\section{References}

1. McDonald DJ, Sim FH, McLeod RA, Dahlin DC. Giantcell tumor of bone, Journal of Bone and Joint Surgery. Series A. 1986; 68(2):235-242.

2. Kundu ZS, Sen R, Dhiman A, Sharma P, Siwach R, Rana P. Effect of intravenous zoledronic acid on histopathology and recurrence after extended curettage in giant cell tumors of bone: A comparative prospective study. Indian Journal of Orthopaedics. 2018; 52(1):45.

3. Szendröi M. Giant-cell tumour of bone. The Journal of bone and joint surgery. British volume. 2004; 86(1):5-12.

4. Goldenberg RR, Campbell CJ, Bonfiglio M. Giantcell tumor of bone. An analysis of two hundred and eighteen cases, Journal of Bone and Joint Surgery. Series A. 1970; 52(4):619-664.

5. Rigollino AV, Fernando TS, Tanaka MH, Souza MM. Giant cell tumor locally advanced around the knee: treatment and literature review. Revista brasileira de ortopedia. 2017; 52(4):473-8.

6. Errani C, Ruggieri P, Asenzio MA, Toscano A, Colangeli S, Rimondi E et al. Giant cell tumor of the extremity: a review of 349 cases from a single institution. Cancer treatment reviews. 2010; 36(1):1-7.

7. Kayias EH, Drosos GI, Anagnostopoulou GA. Resection of the distal ulna for tumours and stabilisation of the stump. A case report and literature review. Acta orthopaedica belgica. 2006; 72(4):484.

8. Dingman PV. Resection of the distal end of the ulna (Darrach operation). An end result study of twenty four cases, The Journal of Bone and Joint Surgery. 1952; 34(4):893-900.

9. Minami A, Ogino T, Minami M. Treatment of distal radioulnar disorders, Journal of Hand Surgery. 1987; 12(2):189-196.

10. Hashizume H, Kawai A, Nishida K, Sasaki K, Inoue H. Ulnar buttress arthroplasty for reconstruction after resection of the distal ulna for giant cell tumour, Journal of Hand Surgery. 1996; 21(2):213-215.

11. Klenke FM, Wenger DE, Inwards CY, Rose PS, Sim FH. Giant cell tumor of bone: risk factors for recurrence. Clinical Orthopaedics and Related Research®. 2011; 469(2):591-9. 
12. Knochentumoren A. Local recurrence of giant cell tumor of bone after intralesional treatment with and without adjuvant therapy. JBJS. 2008; 90(5):1060-7.

13. Baptista AM, de França Camargo AF, Tadeu Caiero M, Seguel Rebolledo DC, Marques Correia LF, Pires de Camargo O. TCG: o que aconteceu após 10 anos de curetagem e cimentação? Estudo restrospectivo de 46 casos. Acta Ortopédica Brasileira, 2014, 22(6).

14. Dahlin DC, Cupps RE, Johnson Jr EW. Giant-cell tumor: A study of 195 cases. Cancer. 1970; 25(5):1061-70.

15. Ferracini R, Masterson EL, Bell RS, Wunder JS. Distal ulnar tumours: results of management by en bloc resection in nine patients and review of the literature. The Journal of Hand Surgery: British \& European Volume. 1998; 23(4):517-21.

16. Roidis NT, Gougoulias NE, Liakou PD, Malizos KN. Distal ulnar implant arthroplasty as a definitive treatment of a recurrent giant-cell tumor. The Journal of hand surgery. 2007; 32(8):1262-6.

17. Chen KH, Wu PK, Chen CF, Chen WM. Zoledronic acidloaded bone cement as a local adjuvant therapy for giant cell tumor of the sacrum after intralesional curettage. European Spine Journal. 2015; 24(10):2182-8.

18. Gouin F, Rochwerger AR, Di Marco A, Rosset P, Bonnevialle P, Fiorenza F, Anract P. Adjuvant treatment with zoledronic acid after extensive curettage for giant cell tumours of bone. European Journal of Cancer. 2014; 50(14):2425-31. 